\title{
Arboviral diseases in the Western Brazilian Amazon: a perspective and analysis from a tertiary health \& research center in Manaus, State of Amazonas
}

\author{
Maria Paula Gomes Mourão[1],[2], Michele de Souza Bastos ${ }^{[1],[2],}$ \\ Regina Maria Pinto de Figueiredo ${ }^{[1]}$, João Bosco de Lima Gimaque ${ }^{[1],[2]}$, \\ Valquíria do Carmo Rodrigues Alves ${ }^{[2],[4]}$, Maria das Graças Gomes Saraiva[ ${ }^{[1],[3]}$, \\ Mário Luis Garcia Figueiredo[1], Rajendranath Ramasawmy ${ }^{[1],[5],}$ \\ Maurício Lacerda Nogueira ${ }^{[1],[6]}$ and Luiz Tadeu Moraes Figueiredo ${ }^{[1],[5]}$
}

[1]. Fundação de Medicina Tropical Dr. Heitor Vieira Dourado, Manaus, AM. [2]. Escola Superior de Ciências da Saúde, Universidade do Estado do Amazonas, Manaus, AM. [3]. Curso de Medicina, Universidade Nilton Lins, Manaus, AM. [4]. Fundação Centro de Controle Oncologia do Estado do Amazonas, Manaus, AM. [5]. Centro de Pesquisa em Virologia, Faculdade de Medicina de Ribeirão Preto, Universidade de São Paulo, Ribeirão Preto, SP. [6]. Faculdade de Medicina de São José do Rio Preto, São José do Rio Preto, SP.

\begin{abstract}
The Fundação de Medicina Tropical Dr. Heitor Vieira Dourado (FMT-HVD), located in Manaus, the capital of the State of Amazonas (Western Brazilian Amazon), is a pioneering institution in this region regarding the syndromic surveillance of acute febrile illness, including arboviral infections. Based on the data from patients at the FMT-HVD, we have detected recurrent outbreaks in Manaus by the four dengue serotypes in the past 15 years, with increasing severity of the disease. This endemicity has culminated in the simultaneous circulation of all four serotypes in 2011, the first time this has been reported in Brazil. Between 1996 and 2009, 42 cases of yellow fever (YF) were registered in the State of Amazonas, and 71.4\% (30/42) were fatal. Since 2010, no cases have been reported. Because the introduction of the yellow fever virus into a large city such as Manaus, which is widely infested by Aedes mosquitoes, may pose a real risk of a yellow fever outbreak, efforts to maintain an appropriate immunization policy for the populace are critical. Manaus has also suffered silent outbreaks of Mayaro and Oropouche fevers lately, most of which were misdiagnosed as dengue fever. The tropical conditions of the State of Amazonas favor the existence of other arboviruses capable of producing human disease. Under this real threat, represented by at least 4 arboviruses producing human infections in Manaus and in other neighboring countries, it is important to develop an efficient public health surveillance strategy, including laboratories that are able to make proper diagnoses of arboviruses.
\end{abstract}

Keywords: Arbovirus. Western Brazilian Amazon. Dengue. Yellow fever. Mayaro fever. Oropouche fever.

\section{INTRODUCTION}

Arboviruses (arthropod-borne viruses) are ribonucleic acid (RNA) viruses (Togaviridae, Flaviviridae, Bunyaviridae, Reoviridae and Rhabdoviridae) that are maintained in nature as zoonoses. Their complex life cycles involve arthropod vectors (mostly mosquitoes and ticks) that transmit these microorganisms by feeding on infected animal blood, mostly birds and mammals. Arboviruses have a great capacity for evolution and adaptation that makes emergence and reemergence of these agents a natural phenomenon ${ }^{(1)}$.

Arbovirus research in Brazil is strongly linked to the Amazon region. Since the beginning of the $20^{\text {th }}$ century, the efforts

Corresponding author: Dra. Maria Paula Gomes Mourão. Lab. Virologia/ FMT-HVD. Av. Pedro Teixeira 25, Dom Pedro, 69040-000 Manaus, AM, Brasil. Phone: 5592 2127-3447; 5592 9142-2474

e-mail: mariapaula.mourao@gmail.com

Received 7 August 2013

Accepted 22 May 2014 of Rockefeller Foundation to understand and control yellow fever have led to the development of a research program in the Amazon region, especially at the Instituto Evandro Chagas (IEC), where field research for the collection of samples from vertebrate reservoirs and mosquitoes has been performed. The history of this field research has been described previously ${ }^{(2)}$.

It is known that hundreds of arboviruses have been isolated in the Amazon Basin, but only a few of these have been related to human disease. These viruses are responsible for more than $95 \%$ of human arboviral diseases reported in the country. The most important arboviruses causing human disease in Brazil are the yellow fever virus (YFV), dengue virus (DENV), Saint Louis encephalitis virus (SLEV), Mayaro virus (MAYV) and Oropouche virus (OROV) ${ }^{(1)}$.

Manaus, located in the middle of the Amazon rain forest, is the capital city of the State of Amazonas and has almost 2 million inhabitants. In 1998, The Fundação de Medicina Tropical Dr. Heitor Vieira Dourado (FMT-HVD) launched in the Amazon region a pioneering investigation of acute febrile 
syndrome, including hemorrhagic fevers. In addition to malaria, arboviral infections by dengue and other arboviruses have been investigated at the institution, which includes a Virology Laboratory qualified for diagnosing arboviral diseases.

\section{DENGUE FEVER}

Manaus has experienced dengue outbreaks since the first one occurred in $1998^{(3)}$. That 1998 outbreak included approximately 23,000 cases caused by DENV1, as reported by the FMT-HVD (Figure 1). In 2001, DENV2 produced a second large outbreak of approximately 9,200 reported cases, including, for the first time in Amazonas State, 67 cases of dengue hemorrhagic fever (Figure 2). No fatalities were observed at the reference center (FMT-HVD) in this period, and more than 200 patients were admitted to the Emergency Department. In October of 2002, DENV3 was detected in Manaus, but since then, there have been only recurrent minor outbreaks during the rainy season (December to April), with an increasing number of severe dengue cases, mostly in children ${ }^{(4)}$. Then, in January of 2008 in Manaus, DENV4 was detected for the first time in Brazil, after a lapse of 26 years $^{(5)}$. Since then, all the dengue serotypes have occurred simultaneously in Manaus.

Following an upward trend of dengue in Brazil, Manaus had a new dengue fever outbreak in the first months of 2011. This outbreak included almost 40,000 cases and it affected patients from all regions of the city, causing diseases of distinct severities. More than 900 acute febrile adult patients were admitted at the FMT-HVD Emergency Department, of which $155(17.2 \%)$ were laboratory-confirmed severe dengue cases, and $158(17.5 \%)$ were presumptive severe dengue cases. All four dengue virus serotypes were observed, the first time simultaneous infections by the four dengue serotypes have been reported in Manaus and in Brazil ${ }^{(6)}$. Two $(0.2 \%)$ fatalities occurred in this period among the febrile hospitalized patients at FMT-HVD, and acute dengue infection could be confirmed in only one. This 2011 outbreak showed the hyperendemicity of DENV in Brazil, where the increased genetic diversity can bring serious consequences, such as an expanded range of pathogenic properties and increased transmissibility and virulence.

Phylogenetic analysis shown that DENV2 from Manaus belongs to the Asian/American genotype II and that DENV3 belongs to genotype III. Both are genotypes that have been circulating throughout Brazil for the last 23 years. In contrast, the DENV4 from Manaus in 2008 belonged to genotype I, which is Asiatic and has never been described on the American continent. DENV4 isolates from Manaus group together with the Philippines/1956/H241 (AY947539) and China_Guangzhou_B5 (AF289029) strains ${ }^{(7)}$. This virus has been in Manaus for several years, producing cases of acute febrile illness but most likely not spreading to other parts of Brazil. In 2008, an Aedes aegypti mosquito infected with genotype I DENV4 was captured in Manaus, supporting the idea that this new virus really was circulating in the city ${ }^{(8)}$. However, after 2010, another DENV4 of genotype II was introduced into Brazil, and it spread throughout Brazil, including into Manaus ${ }^{(9)}$.

In recent years, most likely as a consequence of hyperendemicity, severe dengue disease has become a little more prevalent in children in Amazonas, and atypical cases, such as those having meningoencephalitis, are increasing during dengue outbreaks ${ }^{(10)(11)}$.

\section{YELLOW FEVER}

Historically, YFV was the most important arbovirus disease in the Amazon region. YFV belongs to the Flavivirus genus of Flaviviridae and is widely distributed in tropical Africa and the Americas. Originating in Africa, this virus was imported to Brazil and South America through the slave trade. In South America, YFV caused several outbreaks of urban disease transmitted by Aedes aegypti and it developed a well-adapted sylvatic cycle that involves mosquitoes of the Haemagogus genus and monkeys, both Callitrichiade and Cebidae ${ }^{(1)}$.

The introduction of the very efficient YFV 17D vaccine in the 1930s eradicated the disease in its urban cycle, and yellow fever is now restricted to unvaccinated individuals. In recent

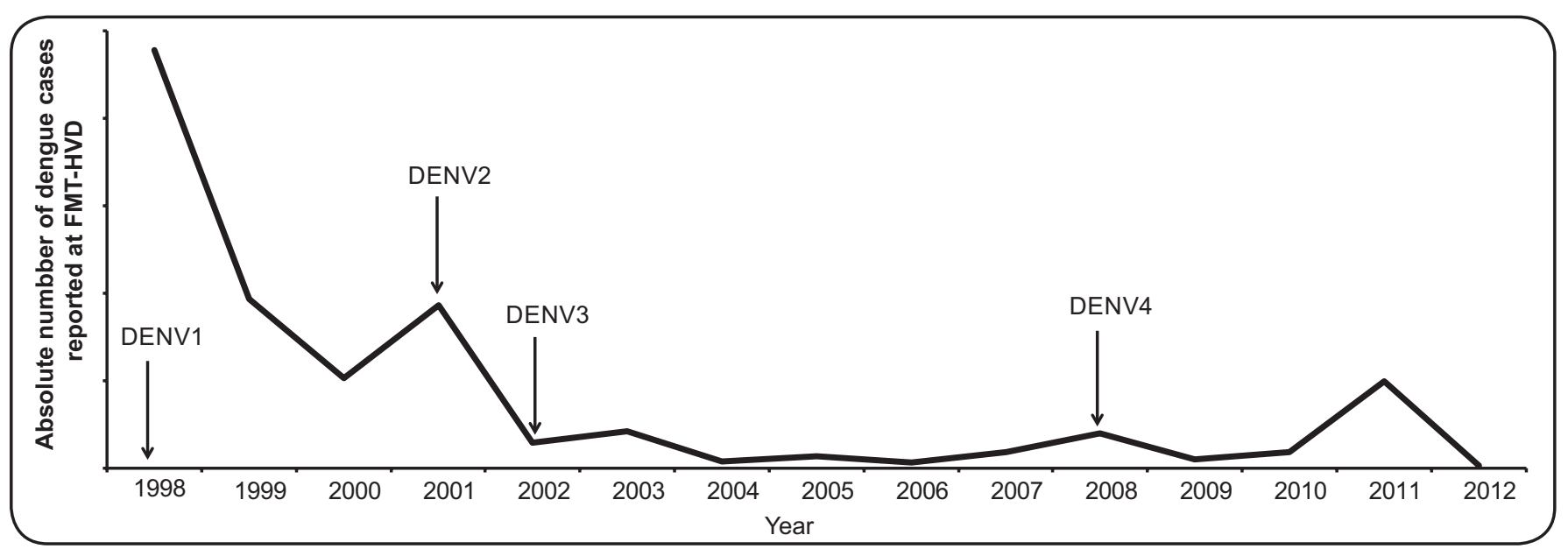

FIGURE 1 - Reported case series of dengue fever at Fundação de Medicina Tropical Dr. Heitor Vieira Dourado (FMT-HVD) (1998-2012) and the first identification DENV serotypes in Manaus, State of Amazonas. DENV: dengue virus. 


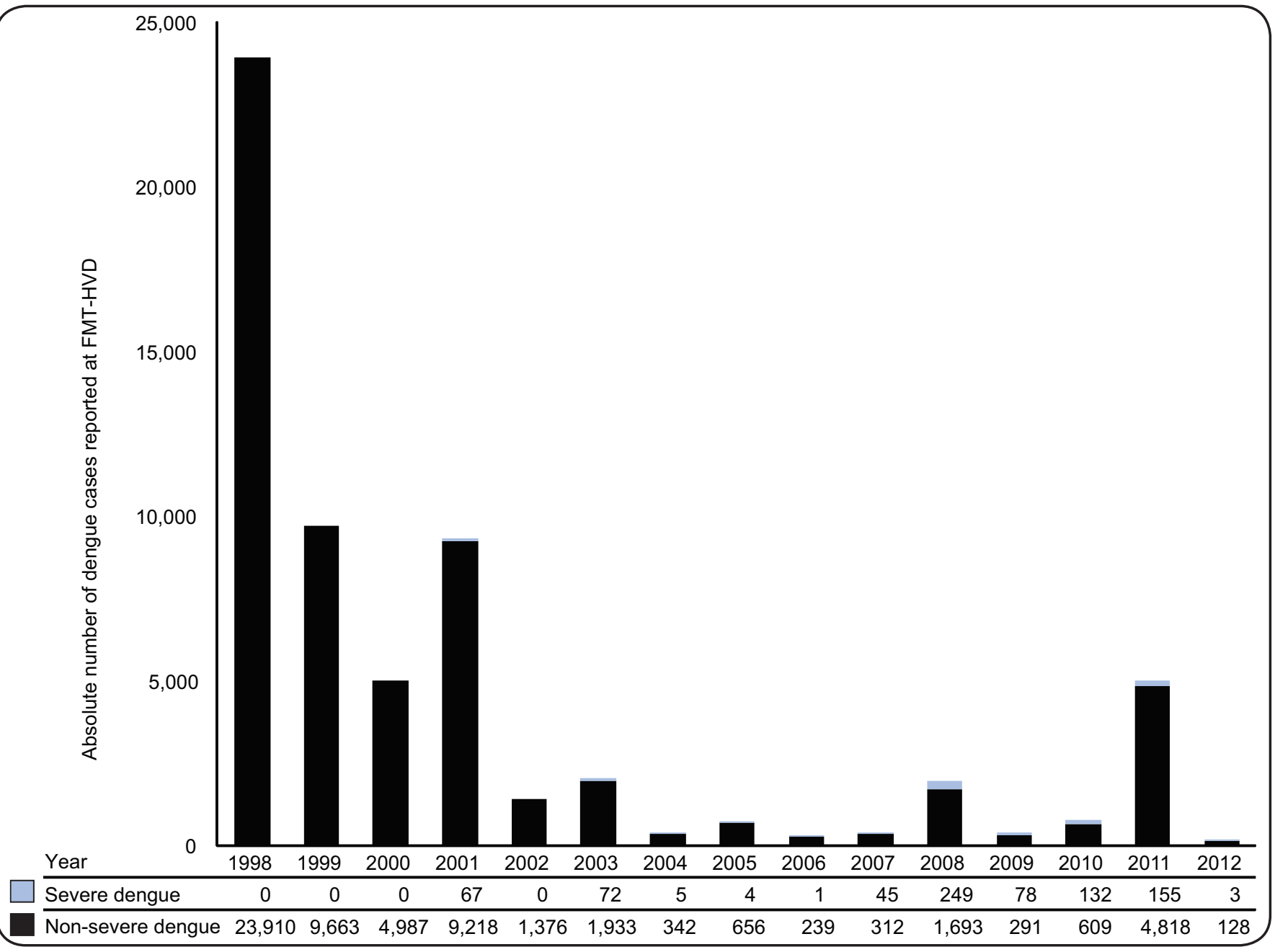

FIGURE 2 - Dengue cases reported at Fundação de Medicina Tropical Dr. Heitor Vieira Dourado (FMT-HVD) (1998-2012), according to severity.

years, an increasing number of cases have emerged with the expansion of tourism in the Amazon region and the Central Plateau of Brazil ${ }^{(12)}$. Additionally, Aedes aegypti is now widely disseminated throughout the country and this could lead to the re-urbanization of yellow fever.

Between 1996 and 2009, 42 cases of yellow fever were registered in the State of Amazonas: $71.4 \%$ (30/42) of the cases were fatal, $26.2 \%(11 / 42)$ were cured and $2.4 \%$ (1/42) were asymptomatic (Figure 3). Of these cases, 98\% (41/42) were autochthonous from 17 municipalities of the State of Amazonas, and one case was imported from the State of Pará. The lethality rate was $71 \%$ (30/42), evidence of a poor surveillance of yellow fever in the state and/or a strong virulence and lack of immunity in affected patients. Of the 30 fatal cases of yellow fever, $10 \%$ $(3 / 30)$ were foreigners ${ }^{(13)}$. The abundance of Aedes aegypti and of Aedes albopictus in rural areas and in Manaus suggests that the high dispersion of these vectors increases the risk of yellow fever outbreaks, and this reinforces the need to ensure high coverage of vaccination and to maintain a continuous and improved epidemiological and entomological surveillance. Haemagogus janthinomys has been found in forest areas approximately $100 \mathrm{~m}$ from residential areas where the vulnerable population lives. These are also areas characterized by the intradomiciliary presence of Aedes aegypti ${ }^{(13)}$.

The accumulated vaccine coverage against yellow fever grew gradually in the State of Amazonas, from 58.9\% in 1996 to $87 \%$ in $2009^{(13)}$. This latest vaccine coverage is still below the acceptable value, i.e., vaccine coverage above $90 \%$ in all municipalities. Also needed is a proof of immunization from all tourists entering the state (airports, ports and road barriers) and a public awareness campaign through all media.

\section{MAYARO FEVER}

Mayaro Alphavirus (MAYV) causes outbreaks of acute febrile illness in the Amazon region. A serologic survey of the population living at the border of Rio Negro river, showed that $139(41.5 \%)$ of 335 participants had antibodies to MAYV. This shows the high level of human infections by MAYV in the State of Amazonas ${ }^{(14)}$.

From January 2007 to December 2008, patients at the FMT-HVD who had more than 5 days of acute febrile illness were systematically tested by a blood smear for malaria and by 


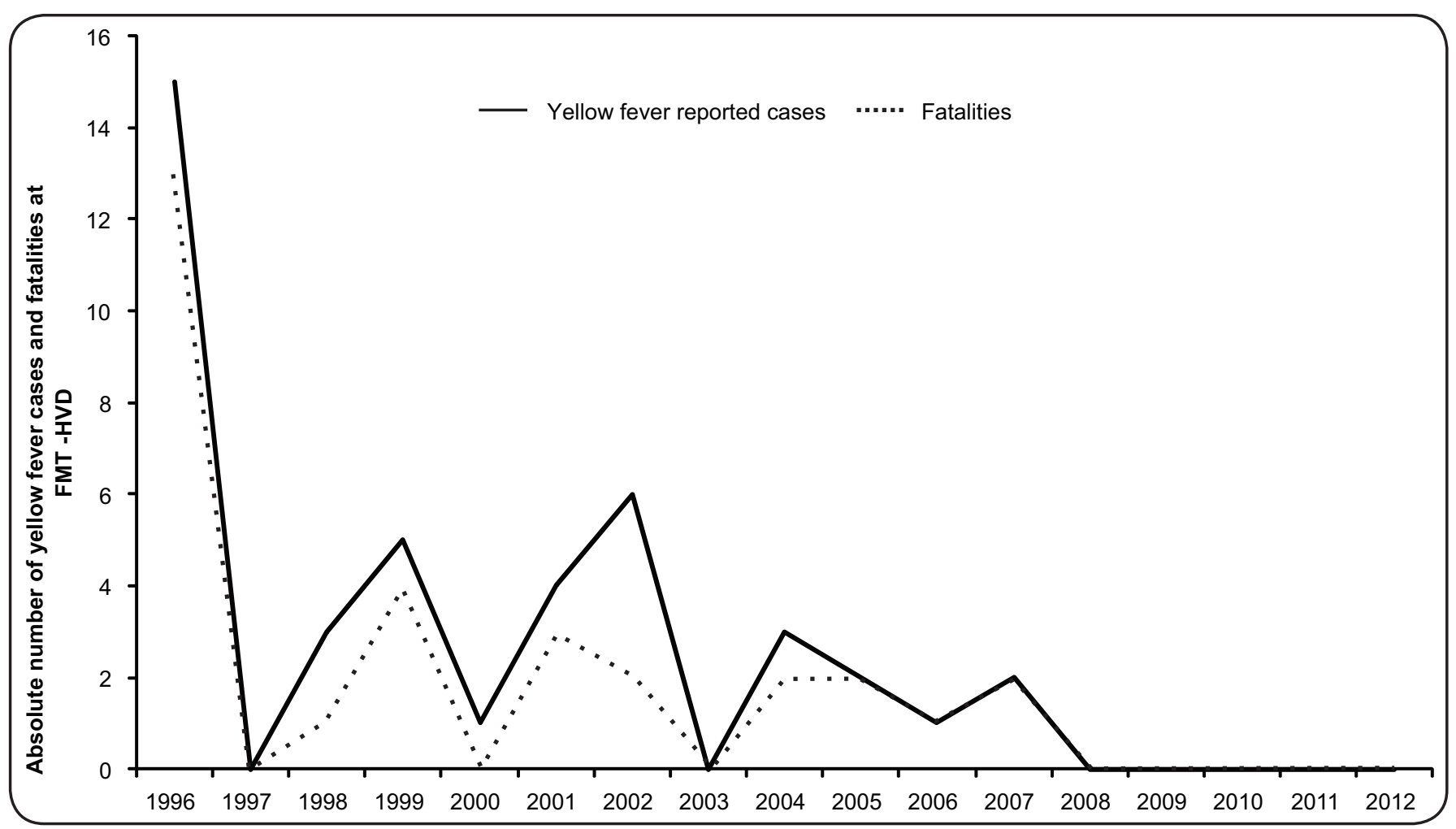

FIGURE 3 - Reported case series and fatalities due to yellow virus at Fundação de Medicina Tropical Dr. Heitor Vieira Dourado (FMT-HVD) (1996-2012).

serology for dengue. Those patients having negative results for both tests were then tested for Mayaro virus. immunoglobulin $\mathrm{M}(\mathrm{IgM})$ antibodies to MAYV were detected in $33(5.2 \%)$ sera of 631 patients tested. Cases of Mayaro fever were observed in both genders and included patients from 6 to 65 years old ${ }^{(15)}$.

The patients with Mayaro fever presented mainly with headache, arthralgia, myalgia and ocular pain. A cutaneous rash was observed in $8(24.2 \%)$ patients and hemorrhagic phenomena were observed in $4(12.1 \%)$. All patients recovered without sequelae and were not hospitalized. It was found that Mayaro fever occurs all year long in Manaus, but the cases are more frequent from November through March, the rainy months of the year ${ }^{(15)}$.

The MAYV genome was detected by reverse transcription polymerase chain reaction (RT-PCR) in the serum of one IgM positive patient. The sequence of 201 nucleotides from the amplicon (AM28725 - Gene Bank submission number HQ664947) showed 97\% homology with the prototype MAYV sequence (TRVL15537), as assessed by Blast ${ }^{(15)}$.

The 33 cases of Mayaro fever reported here are likely to represent the tip of an iceberg, and most likely a much greater number of cases occurred in Manaus during the study period. The disease affects people who are most likely bitten by sylvatic Haemagogus mosquitoes. The transmission of MAYV in a large city such as Manaus is a relevant public health problem, as there is no vaccine for this virus, and the control of sylvatic Haemagogus mosquito vectors is not feasible.

\section{OROPOUCHE FEVER}

Oropouche virus (OROV), a member of the Bunyaviridae family (Orthobunyavirus genus), was first isolated in 1955 in Trinidad. In Brazil, it was first detected in an urban outbreak in Belem in 1961. Since then, dozens of Oropouche fever outbreaks have been described in the Amazon region and in the Central Plateau of Brazil. OROV has an urban and a sylvatic cycle. In the jungle, Aedes and Culex vectors maintain the cycle, but in the urban environment, the Culicoides paraensis is the vector of large outbreaks that can reach up to 100,000 patients. OROV produces a dengue-like, acute febrile illness that can be found in either classical epidemics or as a sporadic disease ${ }^{(16)}$.

Serologic tests for OROV were performed in 631 patients at FMT-HVD and 128 (20.3\%) had IgM antibodies to OROV. The age range was 2-81 years, and $77(60.2 \%)$ were women. As with Mayaro fever, most of the cases occurred during the rainy season from November through March. In addition to fever, patients with Oropouche fever had headache (72.7\%), myalgia (70.3\%), and arthralgia (57.8\%). Rash was observed in $54(42.2 \%)$ patients, and hemorrhagic phenomena (petechiae, epistaxis, and gingival bleeding) were observed in $20(15.5 \%)$ patients. All patients recovered without sequelae and were not hospitalized ${ }^{(17)}$. This outbreak of Oropouche fever in Manaus from 2007 to 2008 was practically unrecognized, and the cases were clinically misdiagnosed as dengue.

A less frequent clinical presentation of OROV infection was also observed in patients at FMT-HVD, after analysis of 110 cerebrospinal fluid (CSF) samples from meningoencephalitis 
syndrome patients. The RNA extracted from CSF was submitted to a reverse transcription-polymerase chain reaction, which revealed the OROV genome in 3 samples, thus confirming that this virus is also a causative of central nervous system (CNS) infection in the State of Amazonas. All 3 patients were adults, and 2 of them had other diseases affecting CNS and immune systems, neurocysticercosis and acquired immunodeficiency syndrome, respectively ${ }^{(18)}$.

Nucleotide sequence analysis shown that the OROV from the CSF of these 3 patients belonged to genotype 1, the same as those previously reported for the virus in the Amazonas ${ }^{(18)}$.

\section{CRITICAL EVALUATION OF THE MAIN CHANGES OBSERVED}

Recently, State of Amazonas has observed a significant reduction of sylvatic yellow fever cases, but a sustained high lethality rate. Furthermore, the continuous appearance of sylvatic cases, despite an immunization policy for all age ranges and the presence of Aedes aegypti and Aedes albopictus in urban and peri-urban areas, makes this region highly vulnerable to yellow fever re-urbanization. Hence, it is necessary to maintain an effective surveillance system, including the monitoring of epizootic cycles in non-human primates, the enhancement of vaccine coverage, prompt human case detection and systematic investigation of contacts.

For dengue fever, Amazonas is experiencing the opposite: an impressive increase of reported cases, with major outbreaks affecting not only the capital but also the largest cities in the countryside. Additionally, with the well-established simultaneous circulation of all four dengue serotypes, there has been an important concentration of severe cases in children, who are still in the process of developing specific acquired immunities to dengue virus.

On the other hand, State of Amazonas has displayed a cumulative and very successful record in outbreak preparedness and clinical management of severe dengue over the past 12 years. This has led to a progressive improvement in organizing the public health system, training health personnel and incorporating new technologies for monitoring vector control and severe cases, such as the use of smartphones for a real-time transmission data.

An impressive example of this is the role of the FMT-HVD. In the 1998 dengue outbreak, the FMT-HVD was responsible for over $60 \%$ of reported cases. In the period since, healthcare for patients suspected to have dengue has been carefully decentralized. This has allowed the FMT-HVD, a tertiary health facility, to dedicate itself mainly to severe dengue cases, which are less than $10 \%$ of reported cases. As a result, State of Amazonas and particularly the FMT-HVD are recognized as having one of the lowest fatality rates for dengue in Brazil.

Another crucial issue for arboviral diseases is diagnostic tools. Indirect diagnosis (IgM/IgG detection), with important limitations due to cross-reaction between similar viral families, is the most widespread test at public health facilities in Brazil. Viral isolation, known as the gold-standard method for classical virologists, also has limitations, mainly due to the short viremia period, the minimum period for growth of 21 days and the low positivity rate. Hence, in the past decade, molecular techniques to diagnose viral diseases have spread and become important because they are, fast, sensitive, specific, reproducible and mostly automated.

The implementation of molecular diagnosis in the past decade has greatly enhanced the syndromic surveillance of febrile diseases by the FMT-HVD, making it a unique sentinel unit in the western Brazilian Amazon. This molecular diagnosis allows systematic monitoring of arboviral circulation in humans, vectors and reservoirs, as well as an opportunity to deliver new diagnostic tests to the population, such as those for viral central nervous system infections.

Physicians and other health professionals working in the Amazon region should be aware that, in addition to malaria and dengue, American native arboviruses produce outbreaks or sporadic human cases and must be included in the differential diagnosis of acute febrile illnesses. These American native arboviruses include the OROV, MAYV, yellow fever, Saint Louis encephalitis (SLEV), Cacipacoré and Rocio viruses.

\section{FUTURE PERSPECTIVES}

The Amazonas state offers perfect conditions for maintenance of arbovirus cycles in nature. This climatic reality, together with an absence of effective measures for controlling Aedes aegypti in Manaus and other cities of the State of Amazonas, strongly suggests that dengue will remain hyperendemic, particularly in Manaus, with alternate or simultaneous circulation of the four viral serotypes. Dengue vaccine, which will most likely become available in the next few years, remains the primary measure for controlling this disease in the Brazilian population, and Manaus and the FMT-HVD will be part of Brazilian studies to prepare and validate the upcoming dengue vaccine.

As mentioned before, it is also important to keep an epidemiological surveillance of primates and mosquitoes to detect the yellow fever virus before human cases appear so that preventive measures and controls can be taken ${ }^{(19)}$.

Mayaro is a zoonotic virus that occurs in the State of Amazonas, and it most likely will produce outbreaks or human sporadic cases in the next years ${ }^{(20)}$. The clinical presentation of Mayaro fever is similar to that described for the other phylogenetically related Alphavirus, Chikungunya virus (CHYKV), including joint manifestations that can be confused with autoimmune diseases ${ }^{1}$. Therefore, it would be important to study the clinical manifestations and the physiopathology of Mayaro fever in our patients. With previous reports of the transmission of MAYV by Aedes mosquitoes, the appearance of this virus in Manaus, which is infested by Aedes aegypti, could generate urban cycles of Mayaro fever involving humans as reservoirs ${ }^{(21)}$.

Oropouche is another arbovirus that occurs in Amazonas and most likely will keep producing outbreaks or human sporadic cases over the coming years. It is known that the number of outbreaks by this virus is increasing in Brazil. However, some important aspects of this arboviral disease remain obscure, such as its virus reservoirs and the clinical presentation of Oropouche 
fever ${ }^{(1)(16)}$. A recurrence of symptoms is described after the first week of fever. The virus can also assault the CNS. Therefore, it would be important to study the clinical manifestations and the physiopathology of Oropouche disease in our patients.

The tropical condition of the State of Amazonas can also favor other arboviruses that are also able to produce human disease. Some of these arboviruses most likely could remain unnoticed in Amazonas and others could be introduced anytime. We mention here SLEV and the Rocio virus (ROCV). SLEV has been isolated in the Amazon region, where studies point to Culex declarator and Culex coronator as vectors, and wild birds, monkeys, sloths, armadillos and marsupials as virus reservoirs ${ }^{(1)}$. In 2006, the first outbreak of SLEV in Brazil was reported. Concomitant with a DENV-3 outbreak in the City of Sao José do Rio Preto, the SLEV genome was detected in clinical samples from 12 patients $^{(22)}$. ROCV caused a human epidemic of severe encephalitis that lasted from 1973 to 1980 in the Ribeira Valley, at the southeastern coast of Brazil. During this outbreak, more than 1,000 cases of encephalitis were reported; the case fatality ratio was $10 \%$, and $30 \%$ of the survivors had neurologic sequelae. After this outbreak, serologic evidence of ROCV circulation in the Ribeira Valley, as well as in other parts of Brazil, has been reported, and public health authorities are concerned about a recurrence of ROCV outbreaks ${ }^{(23)(24)}$.

Among the arboviruses that present the risk of introduction into Brazil and the Amazon region, we emphasize the West Nile virus (WNV) and CHYKV viruses. After WNV was detected in the Western Hemisphere for the first time in 1999, it emerged as a major public health and veterinary problem, as it caused human and equine encephalitis outbreaks, concomitant with extensive mortality in birds, in the northeastern United States of America ${ }^{(25)}$. The virus has spread through North America and towards Latin America ${ }^{(26)}$. There have been recent reports of WNV activity in Colombia, Venezuela and especially Argentina, where anti-WNV antibodies have been detected in birds ${ }^{(26)}$ and the virus was isolated from the brains of horses that had died of encephalitis ${ }^{(27)}$. Human infections by WNV have never been reported in Brazil, but there is serological evidence of WNV activity in the Pantanal region ${ }^{(27)}$. When the sera of 168 horses were tested for flavivirus-reactive antibodies and the positive samples were further tested using a plaque-reduction neutralization test (PRNT90) for WNV, 5 animals had specific virus-neutralizing antibodies, which suggests that this virus has infected horses in Pantanal region. WNV could also be causing undetected infections in the State of Amazonas.

CHYKV is an African virus transmitted to humans by infected mosquitoes, including Aedes aegypti and Aedes albopictus. The huge outbreaks of CHYKV revealed a high number of symptomatic cases, unexpected complications and mother-to-child transmission. Interestingly, the virus produces low lethality in debilitated patients. Imported and autochthonous cases of Chikungunya fever have been recently reported in Brazil, including one confirmed imported casa in the Sate of Amazonas, and there is a real risk of wide introduction and the occurrence of outbreaks by this virus in Brazilian cities infested by Aedes mosquitoes ${ }^{(28)(29)}$.

\section{CONCLUSIONS}

The threat presented by at least 4 different arboviruses that produce human infections (dengue, yellow fever, Mayaro and Oropouche) in Manaus and in other counties of the State of Amazonas makes it extremely important to develop an efficient public health surveillance of arbovirus, with the ability to diagnose and detect severe cases such as dengue hemorrhagic fever, icteric yellow fever and infections of the central nervous system, so that treatment can be given to avoid deaths.

An efficient public health surveillance of arbovirus must include well-equipped virus laboratories operated by trained personnel. The creation of such a structure must be part of a process that requires investment in qualified young virologists and economic support. It is important to highlight that the progress in new virology techniques has been really rapid in the last several years, and some of these modern technologies would be extremely useful in the Amazonas. To achieve this progress, local institutions should cooperate scientifically with universities and research centers from other parts of Brazil and other countries, and steps should be taken to educate graduate students and post-doctoral fellows to develop modern and competent virologists.

Finally, we have shown here that the FMT-HVD, including its Virology Laboratory, has played a key role in performing diagnosis and treatment of patients with arboviral diseases in the State of Amazonas. As previously mentioned, it is necessary for public health authorities to encourage and support the FMTHVD's work with arboviruses. Public health authorities must remember that, even during dengue epidemics, other arboviruses can produce simultaneous outbreaks, as previously seen. It is important to improve surveillance and to decentralize laboratory facilities for the routine diagnosis of arboviral diseases.

\section{CONFLICT OF INTEREST}

The authors declare that there is no conflict of interest.

\section{REFERENCES}

1. Figueiredo LT. Emergent arboviruses in Brazil. Rev Soc Bras Med Trop 2007; 40:224-229.

2. Travassos da Rosa JFS, Travassos da Rosa APA, Vasconcelos PFC, Pinheiro FP, Rodrigues SG, Travassos da Rosa ES, et al. Arboviruses isolated in the Evandro Chagas Institute, including some described for the first time in the Brazilian Amazon region, their known hosts, and their pathology for man. In: Travassos da Rosa APA, Vasconcelos PFC, Travassos da Rosa JFS, editors. An overwiew of arbovirology on Brazil and neighboring countries. Belém: Instituto Evandro Chagas; 1998. p. 19-31.

3. Figueiredo RM, Thatcher BD, Lima ML, Almeida TC, Alecrim WD, Guerra MV. Exanthematous diseases and the first epidemic of dengue to occur in Manaus, Amazonas, State, Brazil, during 19981999. Rev Soc Bras Med Trop 2004; 37:476-479.

4. Rocha LA, Tauil PL. Dengue em criança: aspectos clínicos e epidemiológicos, Manaus, Estado do Amazonas, no período de 2006 e 2007. Rev Soc Bras Med Trop 2009; 42:18-22. 
5. Figueiredo RM, Naveca FG, Bastos MS, Melo MN, Viana SS, Mourão MP, et al. Dengue Virus Type 4, Manaus, Brazil. Emerg Infect Dis 2008; 14:4:667-669.

6. Bastos MS, Figueiredo RMP, Ramasawmy R, Itapirema E, Gimaque JBL, Santos LO, et al. Simultaneous circulation of all four dengue serotypes in Manaus, State of Amazonas, Brazil in 2011. Rev Soc Bras Med Trop 2012; 45:393-394.

7. Melo FL, Romano CM, Andrade Zanotto PM. Introduction of dengue virus 4 (DENV-4) genotype I into Brazil from Asia? PLoS Negl Trop Dis 2009; 3:e390.

8. Figueiredo ML, Alfonso HL, Amarilla AA, Figueiredo LTM, Aquino VH, Costa CA, et al. Detection of dengue serotype 4 in mosquitoes collected in the city of Manaus, Brazil. Virol J 2013; 19:10:60.

9. Acosta PO, Maito RM, Granja F, Cordeiro JS, Siqueira T, Cardoso $\mathrm{MN}$, et al. Dengue virus serotype 4, Roraima State, Brazil. Emerg Infect Dis 2011; 17:1979-1980.

10. Figueiredo LTM. Dengue in Brazil: 1999-2009. Dengue Bullettin (World Health Organization) 2010; 34:6-12.

11. Figueiredo LTM. Dengue in Brazil. Rev Soc Bras Med Trop 2012; 45:285.

12. Ministério da Saúde. Casos de febre amarela, Brasil, Grandes Regiões e Unidades Federadas. 1990-2010. Serviço de Vigilância Sanitária. Brasília: Ministério da Saúde; 2010. (Cited 2013 June). Available at: http://portal.saude.gov.br/portal/arquivos/pdf/tabela_1_fa_2010.pdf

13. Saraiva MGG, Amorim RDS, Moura MAS, Santos ECS, Sampaio LS, Barbosa MGV, et al. Historical analysis of the records of sylvan yellow fever in the State of Amazonas, Brazil, from 1996 to 2009. Rev Soc Bras Med Trop 2013; 46:223-226.

14. Abad-Franch F, Grimmer GH, Paula VS, Figueiredo LTM, Braga WSM, Luz SLB. Emerging Mayaro virus infection in Amazonia: a multimodel inference approach to risk factor assessment. PLoS Negl Trop Dis 2012; 6:e1846.

15. Mourão MP, Bastos MS, Figueiredo RP, Gimaque JB, Galusso ES, Kramer VM, et al. Mayaro fever in Manaus, Brazil, 2007-2008. Vector Borne Zoonotic Dis 2012; 12:42-46.

16. Vasconcelos HB, Azevedo RS, Casseb SM, Nunes-Neto JP, Chiang JO, Cantuária PC, et al. Oropouche fever epidemic in Northern Brazil: Epidemiology and molecular characterization of isolates. J Clin Virol 2009; 44:129-133.

17. Mourão MP, Bastos MS, Gimaqu JB, Mota BR, Souza GS, Grimmer GH, et al. Oropouche fever outbreak, Manaus, Brazil, 2007-2008. Emerg Infect Dis 2009; 15:2063-2064.
18. Bastos MS, Figueiredo LT, Naveca FG, Monte RL, Lessa N, Pinto de Figueiredo RM, et al. Identification of Oropouche Orthobunyavirus in the cerebrospinal fluid of three patients in the Amazonas, Brazil. Am J Trop Med Hyg 2012; 86:732-735.

19. Ministério da Saúde. Fundação Nacional de Saúde. Manual de vigilância epidemiológica de febre amarela. Brasília: Ministério da Saúde; 1999.

20. Azevedo RS, Silva EV, Carvalho VL, Rodrigues SG, Nunes-Neto JP, Monteiro H, et al. Mayaro fever virus, Brazilian Amazon. Emerg Infect Dis 2009; 15:1830-1832.

21. Tesh RB, Watts DM, Russell KL, Damodaran C, Calampa C, Cabezas $\mathrm{C}$, et al. Mayaro virus disease: an emerging mosquitoborne zoonosis in tropical South America. Clin Infect Dis 1999; 28:67-73.

22. Mondini A, Cardeal IL, Lázaro E, Nunes SH, Moreira CC, Rahal P, et al. Saint Louis encephalitis virus, Brazil. Emerg Infect Dis 2001; 13:176-178.

23. Iversson LB, Tiriba AC. Encefalite por arbovírus Rocio. In: Veronesi R, Focaccia R, editors. Tratado de Infectologia. São Paulo: Atheneu; 1996. p. 233-239.

24. Straatmann AA, Santos-Torres S, Vasconcelos PFC, Travassos da Rosa APA, Rodrigues SG, Tavares-Neto J. Evidências sorológicas da circulação do arbovírus Rocio (Flaviviridae) na Bahia. Rev Soc Bras Med Trop 1997; 30:511-515.

25. Centers for Disease Control and Prevention. Outbreak of West Nilelike viral encephalitis-New York. MMWR Morb Mortal Wkly Rep 1999; 48:845-849.

26. Komar N, Clark GG. West Nile virus activity in Latin America and the Caribbean. Rev Panam Salud Publica 2006; 19:112-117.

27. Pauvolid-Corrêa A, Morales MA, Levis S, Figueiredo LT, Couto-Lima D, Campos Z, et al. Neutralising antibodies for West Nile virus in horses from Brazilian Pantanal. Mem Inst Oswaldo Cruz 2011; 106:467-474.

28. Ministério da Saúde. Chikungunya. Serviço de Vigilância Sanitária. Brasília:Ministério da Saúde;2010.(Cited2013 June). Available at: http:// portal.saude.gov.br/portal/arquivos/pdf/clipping_08_09_12_2010. pdf.

29. Ministério da Saúde. Chikungunya Situação atualizada. Serviço de Vigilância em Saúde. Brasília: Ministério da Saúde; 2014. (Cited 2014 November). Available at: http://portalsaude.saude. gov.br/index.php/o-ministerio/principal/secretarias/svs/noticiassvs/15252-saude-atualiza-a-situacao-do-virus-chikungunya 